\title{
RATIONAL APPROXIMATION WITH SERIES
}

D. FENNA

(received 22 July 1960)

The Siegel conjecture on the rational approximation to algebraic numbers was proved a few years ago by $\mathrm{K}$. F. Roth [1] with the following theorem:

Let $\alpha$ be any algebraic number, not rational. If

$$
\left|\alpha-\frac{h}{q}\right|<\frac{1}{q^{\kappa}}
$$

has an infinity of solutions in integers $h$ and $q(q>0)$, then $\kappa \leqq 2$.

This result, which gives a best-possible bound for $\kappa$, improved on earlier results of Liouville, Thue, Siegel, and Dyson.

The analogous problem of approximating to algebraic functions, with degree replacing absolute value, was considered by B. P. Gill [2], who obtained a result corresponding to that of Siegel. In this paper we improve on Gill's result by proving the analogue of Roth's theorem, so obtaining a best-possible result.

Let $\mathfrak{i}$ denote an arbitrary field of zero characteristic and $z$ an indeterminate. Then the set $\Re$ of all formal Laurent series

where

$$
x=\alpha_{d} z^{d}+\alpha_{d-1} z^{d-1}+\cdots,
$$

$$
\alpha_{d}, \alpha_{d-1}, \cdots \in \mathfrak{t},
$$

is a field. Further, the sets $\mathfrak{I}=\mathfrak{l}[z]$ and $\Re=\mathfrak{l}(z)$ form a subring and a subfield of $\Re$ respectively, with

$$
\Re \supseteqq \Re \supseteqq \mathfrak{I} .
$$

If $x \in \Re$ then we denote by $\operatorname{deg} x$ the degree of $x$, i.e.

$$
\begin{aligned}
\operatorname{deg} x & =-\infty \text { if } x \equiv 0, \\
& =d \text { if } \alpha_{d} \text { is the leading non-zero coefficient in } x \neq 0 .
\end{aligned}
$$

The $n$-dimensional space of all vectors $\left(x_{1}, x_{2}, \cdots x_{n}\right)$, where the $x_{k} \in \Re$, is denoted by $\mathfrak{P}_{n}$.

We prove the following theorem. 
THEOREM 1.1. Let $t \in \Re$ be algebraic over $\mathfrak{I}$ but not in $\Re$. If

$$
\operatorname{deg}\left(t-\frac{u}{v}\right)<-v \operatorname{deg} v
$$

for infinitely many $u / v \in \Re$, then $v \leqq 2$.

[Note. It is taken throughout this paper that, in such a representation $u / v$ of an element of $\Re$, the $u, v$ are relatively prime elements of $\mathfrak{T}$.]

This result is clearly best-possible for $v$. For if $d$ is a positive integer there exists a non-trivial set $\alpha_{d}, \alpha_{d-1}, \cdots \alpha_{0}$ of $d+1$ elements of $\mathfrak{l}$ such that, if $v$ is the polynomial

$$
\alpha_{d} z^{d}+\alpha_{d-1} z^{d-1}+\cdots+\alpha_{0},
$$

then the coefficients of $z^{-i}$ in the product $v t$ vanish for $i=1, \cdots d$. Putting $u$ equal to the polynomial part of $v t$ we then have

i.e.

$$
\operatorname{deg}(v t-u)<-d \leqq-\operatorname{deg} v \text {, }
$$

$$
\operatorname{deg}\left(t-\frac{u}{v}\right)<-2 \operatorname{deg} v .
$$

Since $t$ is not rational it follows that, by allowing $d$ to range over all positive integers, we obtain an infinity of distinct solutions $u / v$ of (1.2) with $v=2$.

For the case where the ground field $\mathfrak{f}$ is of positive characteristic $p$ Mahler [3] has shown that the equivalent bound for $v$ is $v \leqq p$, which is again bestpossible.

In the proof of our theorem certain details are omitted because of the essential similarity between our case and that of Roth.

2. Let $x_{1}, \cdots x_{m}$ be $m$ indeterminates and let

$$
F=F\left(x_{1}, \cdots x_{m}\right) \in \mathfrak{R}\left[x_{1}, \cdots x_{m}\right],
$$

i.e. $F$ is a sum of terms of the form

$$
a\left(i_{1}, \cdots i_{m}\right) x_{1}^{i_{1}} \cdots x_{m}^{i_{m}},
$$

where the $a=a\left(i_{1}, \cdots i_{m}\right) \in \Re$. We extend the notation $\operatorname{deg} x$ defined above for $x \in \Re$ to include

$$
\begin{aligned}
\operatorname{deg} F & =-\infty \text { if } F \equiv 0 ; \\
& =\max \left\{\operatorname{deg} a\left(i_{1}, \cdots i_{m}\right)\right\} \text { if } F \not \equiv 0,
\end{aligned}
$$

where the maximum is taken over all non-zero $a$. Clearly this is consistent with our earlier notation, since it merely means that $z^{\text {deg } F}$ is the largest power of $z$ that occurs with non-zero coefficient in $F$. If $F$ itself is in $\Re$ then the two notations agree.

Obviously, if

$$
F^{\prime}\left(x_{1}, \cdots x_{m}\right) \in \Re\left[x_{1}, \cdots x_{m}\right],
$$


then

and

$$
\operatorname{deg}\left(F \pm F^{\prime}\right) \leqq \max \left\{\operatorname{deg} F, \operatorname{deg} F^{\prime}\right\}
$$

$$
\operatorname{deg}\left(F F^{\prime}\right) \leqq \operatorname{deg} F+\operatorname{deg} F^{\prime}
$$

We consider differential operators of the form

$$
\Delta=\frac{\partial^{i_{1}+\cdots+i_{m}}}{\partial x_{1}^{i_{1}} \cdots \partial x_{m}^{i_{m}}}
$$

and call $\left(i_{1}+\cdots+i_{m}\right)$ the order of $\Delta$.

For a positive integer $h$, let

$$
\phi_{\beta}\left(x_{1}, \cdots x_{m}\right) \in \Re\left[x_{1}, \cdots x_{m}\right] \quad(\beta=0,1, \cdots h-1),
$$

and let $\Delta_{\alpha},(\alpha=0,1, \cdots h-1)$, be operators on $x_{1}, \cdots x_{m}$ of order at most $\alpha$. Then we call the determinant

$$
G\left(x_{1}, \cdots x_{m}\right)=\left\{\Delta_{\alpha} \phi_{\beta}\left(x_{1}, \cdots x_{m}\right)\right\}_{\alpha, \beta=0,1, \cdots n-1}
$$

a generalized Wronskian of $\phi_{0}, \phi_{1}, \cdots \phi_{h-1}$.

LEMma 2.1. The necessary and sufficient condition that

$$
\phi_{\beta}\left(x_{1}, \cdots x_{m}\right) \in \mathbb{\Omega}\left[x_{1}, \cdots x_{m}\right] \quad(\beta=0,1, \cdots h-1)
$$

are linearly independent over $\mathfrak{I}$ is that at least one of their generalized Wronskians is non-zero.

LeMma 2.2. Let $R\left(x_{1}, \cdots x_{m}\right)$ be a polynomial in $m \geqq 2$ variables, with coefficients in $\mathfrak{T}$, which is not identically zero. Let $R$ be of degree at most $r_{j}$ in $x_{j},(j=1, \cdots m)$. Then there exists an integer $h$ satisfying

$$
1 \leqq h \leqq r_{m}+1
$$

and there exist differential operators $\Delta_{\lambda},(\lambda=0,1, \cdots h-1)$, on the variables $x_{1}, \cdots x_{m-1}$, of order at most $\lambda$, such that, if

$$
F\left(x_{1}, \cdots x_{m}\right)=\operatorname{det}\left\{\Delta_{\lambda} \frac{\partial^{\mu} R}{\partial x_{m}^{\mu}}\right\}_{\lambda, \mu=0,1, \cdots h-1}
$$

then (i) $F$ has coefficients in $\mathfrak{T}$ and is not identically zero;

$$
F\left(x_{1}, \cdots x_{m}\right)=U\left(x_{1}, \cdots x_{m-1}\right) \cdot V\left(x_{m}\right),
$$

where $U$ and $V$ have coefficients in $\mathfrak{T}, U$ is of degree at most $h r_{j}$ in $x_{j}$, $(j=1, \cdots m-1)$, and $V$ is of degree at most $h r_{m}$ in $x_{m}$.

The proofs of these two lemmas are omitted as they are very similar to those of Roth.

With $F, h$ and $R$ as defined above we prove the following inequality.

Lemma 2.3. $\operatorname{deg} F \leqq h \cdot \operatorname{deg} R$

Proof. Put 


$$
R_{\lambda, \mu}=\Delta_{\lambda} \frac{\partial^{\mu} R}{\partial x_{m}^{\mu}} \quad(\lambda, \mu=0,1, \cdots h-1) .
$$

Now $R$ is the sum of terms of the form

$$
a\left(s_{1}, \cdots s_{m}\right) x_{1}^{s_{1}} \cdots x_{m}^{s_{m}} .
$$

Differentiation with respect to any $x_{1}, \cdots x_{m}$ of such a term will not increase the degree of the coefficient $a\left(s_{1}, \cdots s_{m}\right)$. Hence

$$
\operatorname{deg} R_{\lambda, \mu} \leqq \operatorname{deg} R \quad(\lambda, \mu=0,1, \cdots h-1) .
$$

On the other hand, $F$ is the sum of $h$ ! terms, each of which is a product of the form

$$
\pm R_{\lambda_{0}, 0} R_{\lambda_{1}, 1} \cdots R_{\lambda_{h-1}, h-1} .
$$

It follows that

$$
\operatorname{deg} F \leqq h \cdot \max \left\{\operatorname{deg} R_{\lambda, \mu}\right\},
$$

where the maximum is taken over $\lambda, \mu=0,1, \cdots h-1$. Hence the assertion.

3. Let $P\left(x_{1}, \cdots x_{m}\right) \in \mathfrak{N}\left[x_{1}, \cdots x_{m}\right]$ and, further, let $a_{1}, \cdots a_{m} \in \mathfrak{R}$ and let $r_{1}, \cdots r_{m}$ be any positive numbers.

\section{Definition 3.1.}

The index

$$
\theta\{P\}=\theta\left\{P ;\left(a_{1}, \cdots a_{m}\right) ; r_{1}, \cdots r_{m}\right\}
$$

of $P$ at the point $\left(a_{1}, \cdots a_{m}\right) \in \mathfrak{P}_{m}$ relative to $r_{1}, \cdots r_{m}$ is put equal to $+\infty$ if $P \equiv 0$, otherwise

$$
\theta\{P\}=\min \left\{\frac{j_{1}}{r_{1}}+\cdots+\frac{j_{m}}{r_{m}}\right\}
$$

for all sets of non-negative integers $j_{1}, \cdots j_{m}$ for which

$$
\left\{\frac{\partial^{j_{1}+\cdots+j_{m}} P}{\partial x_{1}^{j_{1}} \cdots \partial x_{m}^{j_{m}}}\right\}_{\left(a_{1}, \cdots a_{m}\right)} \neq 0 \text {. }
$$

The index then has the following properties $\left[Q\left(x_{1}, \cdots x_{m}\right)\right.$ being a second polynomial in $x_{1}, \cdots x_{m}$, and the indices being evaluated at $\left(a_{1}, \cdots a_{m}\right)$ relative to $r_{1}, \cdots r_{m}$.

$$
\begin{gathered}
\theta\{P\} \geqq 0,=0 \text { if and only if } P\left(a_{1}, \cdots a_{m}\right) \neq 0 . \\
\theta\{P+Q\} \geqq \min (\theta\{P\}, \theta\{Q\}) . \\
\varrho\{D ?\}=\vartheta\{P\}+\rho\{Q\}
\end{gathered}
$$

$$
Q=\frac{\partial^{k_{1}+\cdots+k_{m} P}}{\partial x_{1}^{k} \cdots \partial x_{m}^{k_{i}}} \text { for some } k_{1}, \cdots k_{m} \geqq 0,
$$


then

$$
\theta\{Q\} \geqq \theta\{P\}-\left(\frac{k_{1}}{r_{1}}+\cdots+\frac{k_{m}}{r_{m}}\right) .
$$

Also, if $P$ is actually a function of less than $m$ of the variables $x_{1}, \cdots x_{m}$, say $P$ is independent of $x_{m}$, then

$$
\theta\left\{P ;\left(a_{1}, \cdots a_{m}\right) ; r_{1}, \cdots r_{m}\right\}=\theta\left\{P ;\left(a_{1}, \cdots a_{m-1}\right) ; r_{1}, \cdots r_{m-1}\right\} .
$$

Hence, in particular, if $P$ is a function of $x_{1}, \cdots x_{m-1}$ only and $Q$ is a function of $x_{m}$ only, then, from (3.4),

$$
\begin{gathered}
\theta\left\{P Q ;\left(a_{1}, \cdots a_{m}\right) ; r_{1}, \cdots r_{m}\right\} \\
=\theta\left\{P ;\left(a_{1}, \cdots a_{m-1}\right) ; r_{1}, \cdots r_{m-1}\right\}+\theta\left\{Q ;\left(a_{m}\right) ; r_{m}\right\} .
\end{gathered}
$$

4. Let $r_{1}, \cdots r_{m}$ be $m$ positive integers and let $\rho$ be a non-negative number. We denote by

$$
\mathfrak{B}_{m}=\mathfrak{B}_{m}\left(\rho ; r_{1}, \cdots{ }_{m}\right)
$$

the set of all polynomials $R\left(x_{1}, \cdots x_{m}\right) \in \mathfrak{T}\left[x_{1}, \cdots x_{m}\right]$ which satisfy the conditions

(i) $R \not \equiv 0$;

(ii) $R$ is of degree at most $r_{j}$ in $x_{j},(j=1, \cdots m)$;

(iii) $\operatorname{deg} R \leqq \rho$.

Let $v_{1}, \cdots v_{m} \in \mathfrak{I}$ be of positive degree. We put

$$
\Theta_{m}\left\{\rho ; v_{1}, \cdots v_{m} ; r_{1}, \cdots r_{m}\right\}=\sup \theta\left\{R ;\left(\frac{u_{1}}{v_{1}}, \cdots \frac{u_{m}}{v_{m}}\right) ; r_{1}, \cdots r_{m}\right\},
$$

where the supremum is taken over all $R \in \mathfrak{B}_{m}$ and over $u_{1}, \cdots u_{m} \in \mathfrak{I}$ satisfying $\left(u_{i}, v_{i}\right)=1,(i=1, \cdots m)$.

We now obtain an upper bound for $\Theta_{m}$, by induction with respect to $m$. For $m=1$ we have the following inequality.

LEMMA 4.1 .

$$
\Theta_{1}\left\{\rho ; v_{1} ; r_{1}\right\} \leqq \frac{\rho}{r_{1} \cdot \operatorname{deg} v_{1}}
$$

Proof. By the definition of $\theta$, the polynomial $R\left(x_{1}\right)$ is divisible by $\left(x_{1}-u_{1} / v_{1}\right) r_{1} \cdot \theta\{R\}$. Applying Gauss's theorem on factorization, we have

$$
R\left(x_{1}\right)=\left(v_{1} x_{1}-u_{1}\right)^{r_{1} \cdot \theta\{R\}} Q\left(x_{1}\right)
$$

where $Q\left(x_{1}\right) \in \mathfrak{I}\left[x_{1}\right]$. The leading coefficient of $R$ is therefore divisible by $v_{1}^{r_{1} \cdot \theta\{R\}}$, whence

$$
r_{1} \theta\{R\} \operatorname{deg} v_{1} \leqq \operatorname{deg} R \leqq \rho,
$$

and the assertion follows.

LEMmA 4.2. Let $m \geqq 2$ be an integer and let $r_{1}, \cdots r_{m}$ be positive integers 
satisfying

$$
r_{m}>10 \delta^{-1}, \quad r_{j-1}>r_{j} \delta^{-1} \text { for } j=2, \cdots m,
$$

where $0<\delta<1$. Also, let $v_{1}, \cdots v_{m} \dot{\epsilon} \mathfrak{I}$ be of positive degree. Then

$$
\Theta_{m}\left\{\rho ; v_{1}, \cdots v_{m} ; r_{1}, \cdots r_{m}\right\} \leqq 2 \cdot \max \left(\Phi+\Phi^{\frac{1}{2}}+\delta^{\frac{1}{2}}\right)
$$

where the maximum is taken over all integers $h$ satisfying

and where

$$
1 \leqq h \leqq r_{m}+1
$$

$$
\Phi=\Theta_{1}\left\{h \rho ; v_{m} ; h r_{m}\right\}+\Theta_{m-1}\left\{h \rho ; v_{1}, \cdots v_{m-1} ; h r_{1}, \cdots h r_{m-1}\right\} .
$$

We again omit the proof because of its similarity to that of Roth. Note that if

$$
F\left(x_{1}, \cdots, x_{m}\right)=U\left(x_{1}, \cdots, u_{m-1}\right) \cdot V\left(x_{m}\right)
$$

is the function defined in lemma 2.2 then

$$
\max (\operatorname{deg} U, \operatorname{deg} V) \leqq \operatorname{deg} F \leqq h \operatorname{deg} R \leqq h_{\rho} \text {, }
$$

by lemma 2.3. It follows from this and lemma 2.2 that

and

$$
U\left(x_{1}, \cdots x_{m-1}\right) \in \mathfrak{B}_{m-1}\left(h \rho ; h r_{1}, \cdots h r_{m-1}\right)
$$

$$
V\left(x_{m}\right) \in \mathfrak{B}_{1}\left(h_{\rho} ; h r_{m}\right) \text {. }
$$

We now restrict $\delta, v_{1}, \cdots v_{m}, r_{1}, \cdots r_{m}$, give $\rho$ a particular value, and obtain an explicit upper bound for $\Theta_{m}\left\{\rho ; v_{1}, \cdots v_{m} ; r_{1}, \cdots r_{m}\right\}$ in terms of $m$ and $\delta$.

Lemma 4.5. Let $m$ be a positive integer and let $\delta$ satisfy

$$
0<\delta<m^{-1} \text {. }
$$

Let $r_{1}, \cdots r_{m}$ be positive integers satisfying

$$
r_{m}>10 \delta^{-1}, \quad r_{j-1}>r_{j} \delta^{-1} \text { for } j=2, \cdots m \text {. }
$$

Let $v_{1}, \cdots v_{m} \in \mathfrak{I}$ have positive degree and satisfy

$$
r_{j} \operatorname{deg} v_{j} \geqq r_{1} \operatorname{deg} v_{1} \quad(j=2, \cdots m) .
$$

Then

$$
\Theta_{m}\left\{\delta r_{1} \operatorname{deg} v_{1} ; v_{1}, \cdots v_{m} ; r_{1}, \cdots r_{m}\right\}<10^{m} \delta^{\left(\frac{1}{2}\right)^{m}} .
$$

Proof. If $m=1$ then, by lemma 4.1,

$$
\Theta_{1}\left\{\delta r_{1} \operatorname{deg} v_{1} ; v_{1} ; r_{1}\right\} \leqq \frac{\delta r_{1} \operatorname{deg} v_{1}}{r_{1} \operatorname{deg} v_{1}}=\delta<10 \delta^{\frac{1}{2}}
$$

Assume, now, that $m \geqq 2$ and that the lemma holds if $m$ is replaced by $m-1$. Note that the hypothesis remains valid if we replace $m$ ' by $m-1$ 
and $r_{j}$ by $h r_{j},(j=1, \cdots m-1)$. Now, by lemma 4.1,

$$
\Theta_{1}\left\{\delta h r_{1} \operatorname{deg} v_{1} ; v_{m} ; h r_{m}\right\} \leqq \frac{\delta h r_{1} \operatorname{deg} v_{1}}{h r_{m} \operatorname{deg} v_{m}} \leqq \delta,
$$

by (4.6). Hence, if $\Phi$ is the sum defined in (4.4), we have, by the inductive hypothesis,

$$
\Phi<\delta+10^{m-1} \cdot \delta^{\left(\frac{1}{2}\right)^{m-1}}<2\left(10^{m-1} \delta^{\left(\frac{1}{2}\right)^{m-1}}\right) .
$$

Now the hypotheses of lemma 4.2 are less stringent than those of lemma 4.5. Hence lemma 4.2 is applicable and, by (4.3),

$$
\begin{aligned}
\Theta_{m}\left\{\delta r_{1} \operatorname{deg} v_{1} ; v_{1}, \cdots v_{m} ; r_{1}, \cdots r_{m}\right\} \\
<2\left\{2 \cdot 10^{m-1} \delta^{\left(\frac{1}{2}\right)^{m-1}}+2 \frac{1}{2} 10^{(m-1) / 2} \delta^{\left(\frac{1}{2}\right)^{m}}+\delta^{\frac{1}{2}}\right\} \\
\quad<2\left\{\frac{2}{10}+\frac{2^{\frac{1}{2}}}{10}+\frac{1}{10^{2}}\right\} 10^{m} \delta^{\left(\frac{1}{2}\right)^{m}} \\
\quad<10^{m} \delta^{\left(\frac{1}{2}\right)^{m}} .
\end{aligned}
$$

Thus lemma 4.5 holds for $m$ and the induction is complete.

5. Lemma 5.1. Let $n \geqq 2$ and let

and

$$
f(x)=a_{0} x^{n}+a_{1} x^{n-1}+\cdots+a_{n}, \text { where } a_{0} \neq 0,
$$

$$
g(x)=b_{0} x^{s}+b_{1} x^{s-1}+\cdots b_{s}
$$

be two elements of $\mathfrak{T}[x]$, of degree $\alpha$ and $\beta$ in $z$ respectively. Suppose that $d$ is a non-negative integer such that

$$
d \geqq s-n+1
$$

and let $h(x) \in \mathfrak{I}[x]$ be of degree at most $(n-1)$ in $x$ and satisfy

$$
a_{0}^{d} g(x) \equiv h(x), \quad \bmod f(x) .
$$

Then $h(x)$ is of degree at most $(\beta+d \alpha)$ in $z$.

Proof. If $s \leqq n-1$ the lemma is trivial. We complete the proof by induction on $s$.

Assume that $s \geqq n$, whence $d \geqq 1$, and assume that the lemma holds for $(s-1)$ instead of $s$.

Put

$$
g^{*}(x)=a_{0} g(x)-b_{0} x^{-n} f(x) .
$$

Then $g^{*}(x)$ is of degrees at most $(s-1)$ in $x$ and at most $(\beta+\alpha)$ in $z$. Also

$$
a_{0}^{d-1} g^{*}(x) \equiv a_{0}^{d} g(x) \equiv h(x), \quad \bmod f(x) .
$$

Then, by the inductive hypothesis, $h(x)$ is of degree at most

in $z$.

$$
(\beta+\alpha)+(d-1) \alpha=\beta+d \alpha
$$


6. Let $t=t(z) \in \Re$ be algebraic, of degree at least 2 , over $\mathfrak{T}$, and suppose that the inequality (1.2) is satisfied by infinitely many $u / v \in \Re$. Then we wish to show that $v \leqq 2$.

We may assume that $t$ is of negative degree in $z$. For if not, let $t^{\prime}$ be the polynomial part of $t$, and put $t^{*}=t-t^{\prime}$. Then $t^{*}$ is also algebraic and of the same degree as $t$, and is of negative degree in $z$. Further $u / v$ satisfies (1.2) if and only if

$$
\operatorname{deg}\left(t^{*}-\frac{u^{\prime}}{v}\right)<v \operatorname{deg} v
$$

where

$$
u^{\prime}=u-v t^{\prime} \in \mathfrak{T} .
$$

Now $t$ is the root of some irreducible polynomial

$$
f(x)=a_{0} x^{n}+a_{1} x^{n-1}+\cdots+a_{n} \in \mathfrak{I}[x],
$$

where $a_{0} \neq 0, n \geqq 2$. Let $f(x)$ be of degree $\alpha \geqq 0$ in $z$.

We now prove our final lemma.

Let $m$ be a positive integer, and let $\delta, r_{1}, \cdots r_{m}, v_{1}, \cdots v_{m}$ satisfy the following conditions

$$
\begin{array}{ll}
0<\delta<\min \left(m^{-1}, \alpha^{-1}\right), & \\
10^{m} \delta^{(1) m}+2(1+\delta) n m^{\frac{1}{2}}<\frac{1}{2} m, & \\
r_{m}>10 \delta^{-1}, \quad r_{1-1}>r_{j} \delta^{-1} & (j=2, \cdots m), \\
\delta^{2} \operatorname{deg} v_{1}>m, & \\
r_{j} \operatorname{deg} v_{j} \geqq r_{1} \operatorname{deg} v_{1} & (j=2, \cdots m) .
\end{array}
$$

Note that these conditions are stricter than those of lemma 4.5. Define the integer $\rho^{\prime}$ by

whence, by (6.4),

$$
\rho^{\prime} \leqq \delta r_{1} \operatorname{deg} v_{1}<\rho^{\prime}+1,
$$

$$
\rho^{\prime}+1>\delta^{-1} r_{1} m .
$$

Define the numbers $\lambda, \gamma, \eta$ by

$$
\begin{aligned}
& \lambda=4(1+\delta) n m^{t} \\
& \gamma=\frac{1}{2}(m-\lambda) \\
& \eta=10^{m} \delta^{(\mathbf{t})^{m}}
\end{aligned}
$$

Note that $(6.2)$. is then equivalent to

$$
\eta<\gamma \text {. }
$$

LEMMA 6.10. Suppose that the conditions (6.1)-(6.5) are satisfied, and suppose that $u_{1}, \cdots u_{m} \in \mathfrak{I}$ are relatively prime to $v_{1}, \cdots v_{m}$ respectively. Then there exists a polynomial 


$$
Q\left(x_{1}, \cdots x_{m}\right) \in \mathfrak{T}\left[x_{1}, \cdots x_{m}\right]
$$

of degree at most $r_{j}$ in $x_{j},(j=1, \cdots m)$, such that

(i) $\theta\left\{Q ;(t, \cdots t) ; r_{1}, \cdots r_{m}\right\} \geqq \gamma-\eta$;

(ii) $Q\left(\frac{u_{1}}{v_{1}}, \cdots \frac{u_{m}}{v_{m}}\right) \neq 0$;

(iii) for all

$$
Q_{i_{1}, \cdots i_{m}}\left(x_{1}, \cdots x_{m}\right)=\frac{\partial^{i_{1}+\cdots+i_{m}}}{\partial x_{1}^{i_{1}} \cdots \partial x_{m}^{i_{m}}} Q
$$

where $i_{1}, \cdots i_{m}$ are non-negative integers,

$$
Q_{i_{1}}, \cdots i_{m}(t, \cdots t)
$$

is of degree at most $\rho^{\prime}$ in $z$.

Proof. We consider polynomials $W\left(x_{1}, \cdots x_{m}\right) \in \mathfrak{I}\left[x_{1}, \cdots x_{m}\right]$ of the form

$$
W\left(x_{1}, \cdots x_{m}\right)=\sum_{d_{0}=0}^{\rho^{0}} \sum_{d_{1}=0}^{r_{1}} \cdots \sum_{d_{m}=0}^{r_{m}} \xi\left(d_{0}, d_{1}, \cdots d_{m}\right) z^{d_{0}} x_{1}^{d_{1}} \cdots x_{m}^{d_{m}} .
$$

Here the total number of coefficients $\xi\left(d_{0}, d_{1}, \cdots d_{m}\right) \in \mathfrak{t}$ is

$$
\left(\rho^{\prime}+1\right)\left(r_{1}+1\right) \cdots\left(r_{m}+1\right),=M \text { say. }
$$

Denote by $j^{(i)},(i=1, \cdots D)$, the $D$ sets of integers $j_{1}, \cdots j_{m}$ satisfying

$$
0 \leqq j_{1} \leqq r_{1}, \cdots 0 \leqq j_{m} \leqq r_{m} \text { and } \frac{j_{1}}{r_{1}}+\cdots+\frac{j_{m}}{r_{m}} \leqq \frac{1}{2}(m-\lambda) \text {. }
$$

By a result of Roth, ([1], lemma 8)

$$
\begin{aligned}
D & \leqq 2 m^{\frac{1}{2}} \lambda^{-1}\left(r_{1}+1\right) \cdots\left(r_{m}+1\right), \\
& =2 m^{\frac{1}{2}} \lambda^{-1}\left(\rho^{\prime}+1\right)^{-1} M \text { by }(6.12) .
\end{aligned}
$$

For $i=1, \cdots D$, put

$$
W_{g^{(i)}}\left(x_{1}, \cdots x_{m}\right)=\frac{\partial^{j_{1}+\cdots+y_{m}} W}{\partial x_{1}^{j_{2}} \cdots \partial x_{m}^{j_{m}}},
$$

where $j^{(i)}=\left(j_{1}, \cdots j_{m}\right)$. Then, for each such derivative, we form the polynomial

$$
W_{j^{(1)}}(x, \cdots x) \in \mathfrak{T}[x],
$$

which is of degree at most $\left(r_{1}+\cdots+r_{m}\right), \leqq m r_{1}$, in $x$ and, also, of degree at most $\rho^{\prime}$ in $z$.

Now, let

$$
T_{\text {g(x) }}(W ; x) \in \mathfrak{T}[x]
$$

be that element, of order at most $n-1$ in $x$, which satisfies

$$
a_{0}^{m r_{1}} W_{j^{(s)}}(x, \cdots x) \equiv T_{j(t)}(W ; x), \quad \bmod f(x) .
$$


Since $m r_{1} \geqq \max \left\{0,\left(m r_{1}-n+1\right)\right\}$, we have, by lemma 5.1 ,

$$
\operatorname{deg} T_{j^{(b)}} \leqq \rho^{\prime}+m r_{1} \alpha .
$$

Hence, for a given $j^{(i)}$, the polynomial $T_{j(s)}(W ; x)$ is defined by at most

elements of $\mathfrak{l}$.

$$
n\left(\rho^{\prime}+m r_{1} \alpha+1\right)
$$

Therefore, for each $W$, the set $T_{y^{(n)}}(W ; x),(i=1, \cdots D)$, is defined by at most

$$
D n\left(\rho^{\prime}+m r_{1} \alpha+1\right)
$$

elements of $\mathfrak{t}$. Obviously these elements are combinations of the $\xi\left(d_{0}, d_{1}, \cdots\right.$ $\left.d_{m}\right)$, the integers, and the known elements of $f$ involved in $f(x)$. However, they are linear and homogeneous in the unknowns $\xi\left(d_{0}, d_{1}, \cdots d_{m}\right)$ occurring in $W$. But

$$
\begin{aligned}
D n\left(\rho^{\prime}+m r_{1} \alpha+1\right) \leqq 2 m \frac{1}{2} & M n \lambda^{-1}\left(\frac{\rho^{\prime}+m r_{1} \alpha+1}{\rho^{\prime}+1}\right) \text { by }(6.13), \\
& \leqq \frac{M}{2(1+\delta)}\left(1+\frac{m r_{1} \alpha}{\rho^{\prime}+1}\right) \text { by }(6.7), \\
& <M \text { by }(6.1) \text { and }(6.6) .
\end{aligned}
$$

It follows that $W$ may be chosen so that

$$
T_{j^{(w)}}(W ; x) \equiv 0, \quad \bmod f(x) \quad(i=1, \cdots D) .
$$

Since $a_{0} \neq 0$ we then have

$$
W_{j(1)}(x, \cdots x) \equiv 0, \quad \bmod f(x) \quad(i=1, \cdots D)
$$

and, since $f(t)=0$ by definition of $f$, the derivatives $W_{j(1)}\left(x_{1}, \cdots x_{m}\right)$ satisfy

Hence

$$
W_{j(1)}(t, \cdots t)=0 \quad(i=1, \cdots D) .
$$

Now, also,

$$
\begin{gathered}
\theta\left\{W ; t, \cdots t ; r_{1}, \cdots r_{m}\right\} \geqq \frac{1}{2}(m-\lambda) \\
=\gamma \text { by }(6.8) .
\end{gathered}
$$

$$
W\left(x_{1}, \cdots x_{m}\right) \in \mathfrak{B}_{m}\left(\delta r_{1} \operatorname{deg} v_{1} ; r_{1}, \cdots r_{m}\right) .
$$

By lemma 4.5

$$
\theta\left\{W ;\left(\frac{u_{1}}{v_{1}}, \cdots \frac{u_{m}}{u_{m}}\right) ; r_{1}, \cdots r_{m}\right\}<\eta
$$

where $\eta$ is defined in (6.9). Hence, there exists non-negative integers $k_{1}, \cdots k_{m}$ such that

$$
\frac{k_{1}}{r_{1}}+\cdots+\frac{k_{m}}{r_{m}}<\eta
$$


and if

then

$$
Q\left(x_{1}, \cdots x_{m}\right)=\frac{\partial^{k_{1}+\cdots+k_{m}} W}{\partial x_{1}^{k_{1}} \cdots \partial x_{m}^{k_{m}}}
$$

$$
Q\left(\frac{u_{1}}{v_{1}}, \cdots \frac{u_{m}}{v_{m}}\right) \neq 0
$$

Then, by (3.5),

$$
\theta\left\{Q ;(t, \cdots t) ; r_{1}, \cdots r_{m}\right\} \geqq \gamma-\eta
$$

and so $Q$ satisfies parts (i) and (ii) of the lemma.

It also satisfies part (iii). For both $Q\left(x_{1}, \cdots x_{m}\right)$ and the derivative $Q_{i_{1}, \cdots i_{m}}$ defined in $(6.11)$ are clearly elements of $\mathfrak{T}\left[x_{1}, \cdots x_{m}\right]$ of degree at most $\rho^{\prime}$ in $z$. Then, since $t$ is of negative degree,

$$
\operatorname{deg} Q_{i_{1}, \cdots i_{m}}(t, \cdots t) \leqq \rho^{\prime} .
$$

This completes the proof of lemma 6.10.

7. Proof of Theorem 1.1. We suppose that $v>2$ and that the inequality

$$
\operatorname{deg}\left(t-\frac{u}{v}\right)<-v \operatorname{deg} v
$$

has infinitely many solutions $u / v \in \Re$.

We can show (after Gill [2]) that for any integer $\mu \geqq 0$ there is at most one solution $u / v$ of (7.1) for which $\operatorname{deg} v=\mu$. For suppose that $r / s$ is also a solution, with $\operatorname{deg} s=\mu$. Then (7.1) implies

$$
\operatorname{deg}(s u-r v)<-v \mu+\operatorname{deg} s+\operatorname{deg} v=\mu(2-v) \leqq 0
$$

since $\mu \geqq 0$ and $v>2$. But $r, s, u, v \in \mathfrak{T}$ whence $s u-r v \in \mathfrak{T}$ and so is identically zero. Since $s, v \neq 0$ this implies that $r / s$ and $u / v$ are identical.

From this it follows that an infinity of solutions of (7.1) implies solutions for which $\operatorname{deg} v$ is arbitrarily large. We deduce a contradiction of this.

We first choose $m$ so that

$$
m>4 n m t \text {, and } 2 m\left(m-4 n m^{1}\right)^{-1}>v .
$$

If $\delta$ is sufficiently small we then have

$$
m-4(1+\delta) n m^{1}-2 \eta<0,
$$

which is the same as (6.2). We choose $\delta$ to satisfy also the inequality (6.1) and further to satisfy

$$
\frac{2 m(1+2 \delta)}{m-4(1+\delta) n m^{2}-2 \eta}<v .
$$

This inequality is equivalent to 


$$
\frac{m(1+2 \delta)}{\gamma-\eta}<v .
$$

Now let $u_{1} / v_{1}$ be a solution of (7.1), with $\left(u_{1}, v_{1}\right)=1$, and so that $v_{1}$ satisfies (6.4). Let $u_{2} / v_{2}, \cdots u_{m} / v_{m}$ be further solutions of $(7.1)$ with $\left(u_{i}, v_{i}\right)=1$, $(i=2, \cdots m)$, such that

$$
\operatorname{deg} v_{j}>2 \delta^{-1} \operatorname{deg} v_{j-1}
$$$$
(j=2, \cdots m) .
$$

Now take $r_{1}$ to be an integer satisfying

$$
r_{1} \operatorname{deg} v_{1}>10 \delta^{-1} \operatorname{deg} v_{m},
$$

and define $r_{2}, \cdots r_{m}$ by

$$
\frac{r_{1} \operatorname{deg} v_{1}}{\operatorname{deg} v_{j}} \leqq r_{j}<1+\frac{r_{1} \operatorname{deg} v_{1}}{\operatorname{deg} v_{j}} \quad(j=2, \cdots m) .
$$

Then (6.5) is satisfied. Also

$$
\frac{r_{j} \operatorname{deg} v_{j}}{r_{1} \operatorname{deg} v_{1}}<1+\frac{\operatorname{deg} v_{j}}{r_{1} \operatorname{deg} v_{1}} \leqq 1+\frac{\operatorname{deg} v_{m}}{r_{1} \operatorname{deg} v_{1}}<1+\frac{\delta}{10}<1+\delta .
$$

The conditions (6.3) are satisfied, since

and

$$
r_{m} \geqq \frac{r_{1} \operatorname{deg} v_{1}}{\operatorname{deg} v_{m}}>10 \delta^{-1}
$$

$$
\frac{r_{j-1}}{r_{j}}>\frac{\operatorname{deg} v_{j}}{\operatorname{deg} v_{j-1}}\left(1+\frac{\delta}{10}\right)^{-1}>\delta^{-1} .
$$

Now let $Q\left(x_{1}, \cdots x_{m}\right) \in \mathfrak{T}\left[x_{1}, \cdots x_{m}\right]$ be the polynomial of lemma 6.10.

Since $Q$ is of degree at most $r_{j}$ in $x_{j},(j=1, \cdots m)$, and is non-zero for $x_{i}=u_{i} / v_{i},(i=1, \cdots m)$, we have

$$
v_{1}^{r_{1}} \cdots v_{m}^{r_{m}} Q\left(\frac{u_{1}}{v_{1}}, \cdots \frac{u_{m}}{v_{m}}\right) \in \mathfrak{T}, \neq 0
$$

Thus,

(7.5) $\operatorname{deg} Q\left(\frac{u_{1}}{v_{1}}, \cdots \frac{u_{m}}{v_{m}}\right) \geqq-\left(r_{1} \operatorname{deg} v_{1}+\cdots+r_{m} \operatorname{deg} v_{m}\right) \geqq-m r_{1}(1+\delta) \operatorname{deg} v_{1}$, by (7.4). On the other hand,

$$
Q\left(\frac{u_{1}}{v_{1}}, \cdots \frac{u_{m}}{v_{m}}\right)=\sum_{i_{1}=0}^{r_{1}} \cdots \sum_{i_{m}=0}^{r_{m}} Q_{i_{1}} \cdots i_{m}(t, \cdots t) \cdot\left(\frac{u_{1}}{v_{1}}-t\right)^{i_{1}} \cdots\left(\frac{u_{m}}{v_{m}}-t\right)^{i_{m}}
$$

where, by (i) of lemma 6.10 , the terms with

$$
\frac{i_{1}}{r_{1}}+\cdots+\frac{i_{m}}{r_{m}}<\gamma-\eta
$$


vanish. In every other term we have

$$
\begin{aligned}
\operatorname{deg}\left(\left(\frac{u_{1}}{v_{1}}-t\right)^{i_{1}} \cdots\left(\frac{u_{m}}{v_{m}}-t\right)^{i_{m}}\right) & <-v\left(i_{1} \operatorname{deg} v_{1}+\cdots+i_{m} \operatorname{deg} v_{m}\right) \\
& \leqq-v r_{1}(\gamma-\eta) \operatorname{deg} v_{1}, \text { by }(7.3) .
\end{aligned}
$$

By (iii) of lemma 6.10 , it follows that

$$
\begin{aligned}
\operatorname{deg} Q\left(\frac{u_{1}}{v_{1}}, \cdots \frac{u_{m}}{v_{m}}\right) & \leqq \rho^{\prime}-v r_{1}(\gamma-\eta) \operatorname{deg} v_{1} \\
& \leqq \delta r_{1} \operatorname{deg} v_{1}-v r_{1}(\gamma-\eta) \operatorname{deg} v_{1} .
\end{aligned}
$$

Comparing this with (7.5) we have

$$
\begin{aligned}
v r_{1}(\gamma-\eta) \operatorname{deg} v_{1} & \leqq \delta r_{1} \operatorname{deg} v_{1}+(1+\delta) m r_{1} \operatorname{deg} v_{1}, \\
& <m(1+2 \delta) r_{1} \operatorname{deg} v_{1}
\end{aligned}
$$

since $m \geqq 2$. Now $\operatorname{deg} v_{1} \neq 0$, hence

$$
v<\frac{m(1+2 \delta)}{\gamma-\eta}
$$

contrary to (7.2), and the proof is complete.

This work was carried out under the auspices of the U.K. Department of Scientific and Industrial Research, and the guidance of Professor K. Mahler F.R.S., to both of whom the author is indebted.

\section{References}

[1] Roth, K. F., Rational approximations to algebraic numbers. Mathematika, Vol. 2, p. 1.

[2] Gill, B. P., An analogue for algebraic functions of the Thue-Siegel theorem. Annals of Maths., Ser. 2, Vol. 31, p. 207.

[3] Mahler, K., On a theorem of Liouville in fields of positive characteristic. Canadian Journal of Maths., Vol. 1, p. 397.

Department of Defence, Canberra. 